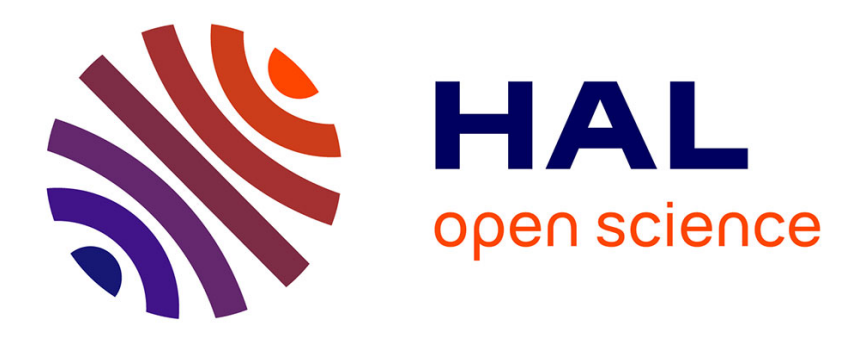

\title{
Generalized Euclidean Distances for Elasticity Tensors
} Leo Morin, Pierre Gilormini, Katell Derrien

\section{To cite this version:}

Leo Morin, Pierre Gilormini, Katell Derrien. Generalized Euclidean Distances for Elasticity Tensors. Journal of Elasticity, 2020, 138 (2), pp.221-232. 10.1007/s10659-019-09741-z . hal-02535683

\section{HAL Id: hal-02535683 \\ https://hal.science/hal-02535683}

Submitted on 7 Apr 2020

HAL is a multi-disciplinary open access archive for the deposit and dissemination of scientific research documents, whether they are published or not. The documents may come from teaching and research institutions in France or abroad, or from public or private research centers.
L'archive ouverte pluridisciplinaire HAL, est destinée au dépôt et à la diffusion de documents scientifiques de niveau recherche, publiés ou non, émanant des établissements d'enseignement et de recherche français ou étrangers, des laboratoires publics ou privés. 


\title{
Generalized Euclidean Distances for Elasticity Tensors
}

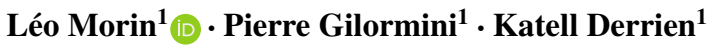

\begin{abstract}
The aim of this short paper is to provide, for elasticity tensors, generalized Euclidean distances that preserve the property of invariance by inversion. First, the elasticity law is expressed under a non-dimensional form by means of a gauge, which leads to an expression of elasticity (stiffness or compliance) tensors without units. Based on the difference between functions of the dimensionless tensors, generalized Euclidean distances are then introduced. A subclass of functions is proposed, which permits the retrieval of the classical log-Euclidean distance and the derivation of new distances, namely the arctan-Euclidean and power-Euclidean distances. Finally, these distances are applied to the determination of the closest isotropic tensor to a given elasticity tensor.
\end{abstract}

Mathematics Subject Classification 74B05 $\cdot 74 \mathrm{E} 10 \cdot 15 \mathrm{~A} 69 \cdot 15 \mathrm{~B} 48$

Keywords Elasticity tensor $\cdot \log$-Euclidean distance $\cdot$ Closest isotropic tensor

\section{Introduction}

Fourth-order tensors are mathematical objects that play crucial roles in several branches of physics such as continuum mechanics [1,2] and magnetic resonance imaging [3]. In continuum mechanics, fourth-order tensors naturally arise in the elasticity law which describes, at smalls strains, the elastic behavior of crystalline materials. The determination of an elasticity tensor, which can be performed from mechanical tensile tests [4], acoustic measurements

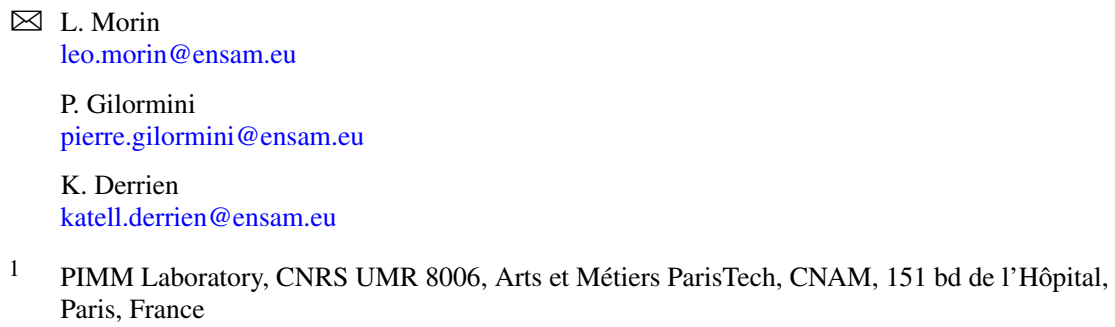


[5] or ab initio calculations [6], leads a priori to 21 constants in the general case of a triclinic material.

In many applications, it is of interest to reduce an arbitrary set of 21 elastic constants to a smaller set, which would correspond to higher symmetries of the elasticity tensor. In particular, it is often useful to start with an isotropic behavior for preliminary calculations of complex structures. It is thus of interest to provide the closest isotropic tensor to a given arbitrary elasticity tensor. Gazis et al. [7] were the first to investigate the determination of the closest isotropic tensor to a given elasticity tensor, by means of an Euclidean projection. However, the closest isotropic elasticity tensor defined with this Euclidean norm is not unique and depends on the choice of stiffness or compliance to express elasticity [8]. This drawback is due to the fact that the Euclidean distance between tensors is not invariant under inversion of the elasticity tensor. To address this, alternative distance functions that preserve the property of invariance under inversion have been proposed. The Riemannian function of Moakher [9] and the log-Euclidean function of Arsigny et al. [10], which are invariant under inversion, have notably been applied to the determination of the closest isotropic tensor to an arbitrary tensor [8, 11-13].

The aim of this work is to generalize the previous Euclidean [7] and log-Euclidean [10] distances with new distances that preserve the property of invariance by inversion, in order to investigate the determination of the closest isotropic tensor to an arbitrary tensor [8] in a general case. The paper is organized as follows. The $f$-Euclidean distance is introduced in the scalar case in Sect. 2, as a generalization of the Euclidean distance for arbitrary functions invariant by inversion. Section 3 extends the scalar $f$-Euclidean distance to fourth-order positive-definite tensors. In Sect. 4, closed-form expressions of functions that achieve invariance by inversion are derived, which lead to new distances, namely the arctan-Euclidean and the power-Euclidean distances. Finally, the determination of the closest isotropic tensor to an arbitrary elasticity tensor is investigated in Sect. 5 for these new distance functions.

\section{The $f$-Euclidean Distance for Elasticity Tensors: The Scalar Case}

We begin with the scalar case of the elasticity law which will shed light on how to tackle the tensorial case; it will notably allow the determination of the properties of scalar functions $f$ that define the $f$-Euclidean distance.

\subsection{Preliminaries}

The constitutive relation in one-dimensional linear elasticity is given by the classical Hooke's law, which establishes a linear relation between the uniaxial stress $\sigma$ and strain $\varepsilon$,

$$
\sigma=E \varepsilon, \quad \varepsilon=\frac{1}{E} \sigma
$$

where $E$ is Young's modulus. The stored elastic energy per unit length $w$ is classically given by

$$
w=\frac{1}{2} \sigma \varepsilon=\frac{1}{2} E \varepsilon^{2}=\frac{1}{2} \frac{1}{E} \sigma^{2} .
$$

Our objective is to derive a distance function between two materials, characterized by their stiffnesses that correspond to Young's moduli $E_{1}$ and $E_{2}$, or their compliances that correspond to the inverses of Young's moduli, $1 / E_{1}$ and $1 / E_{2}$, which verifies the following properties: 
- First and foremost, the defining properties of a distance function must be checked [14], $\forall E_{1}, E_{2}, E_{3} \in \mathbb{R}^{>0}$ (where $\mathbb{R}^{>0}$ denotes the set of strictly positive real numbers):

1. It is nonnegative:

$$
d\left(E_{1}, E_{2}\right) \geq 0
$$

2. It verifies the identity of indiscernibles:

$$
d\left(E_{1}, E_{2}\right)=0, \quad \text { if and only if } E_{1}=E_{2} .
$$

3. It is symmetric in the arguments:

$$
d\left(E_{1}, E_{2}\right)=d\left(E_{2}, E_{1}\right)
$$

4. It respects the triangle inequality:

$$
d\left(E_{1}, E_{2}\right) \leq d\left(E_{1}, E_{3}\right)+d\left(E_{2}, E_{3}\right) .
$$

- Then, the distance function must be invariant under the operation of inversion in order not to favor the behavior law expressed in stiffness or in compliance. This leads, in the scalar case, to the following property

$$
d\left(E_{1}, E_{2}\right)=d\left(1 / E_{1}, 1 / E_{2}\right)
$$

\subsection{The Classical Euclidean Distances}

The simplest distance between elastic moduli is based on the classical Euclidean metric, as the result of some energetics reasoning.

Primal Euclidean Distance The most classical Euclidean distance for elasticity law, which is called the primal Euclidean distance, is obtained from the difference between the elastic energy stored by two different materials for an imposed loading $\operatorname{strain} \varepsilon_{0}$. In that case, the stress states read

$$
\sigma_{1}=E_{1} \varepsilon_{0}, \quad \sigma_{2}=E_{2} \varepsilon_{0},
$$

and the difference between stored elastic energies is given by

$$
w_{1}-w_{2}=\frac{1}{2}\left(\sigma_{1}-\sigma_{2}\right) \varepsilon_{0}=\frac{1}{2}\left(E_{1}-E_{2}\right) \varepsilon_{0}^{2} .
$$

The distance between the two elastic behaviors is given by the primal Euclidean distance, expressed in terms of the stiffnesses $E_{1}$ and $E_{2}$ :

$$
d_{\text {primal }}\left(E_{1}, E_{2}\right)=\left|E_{1}-E_{2}\right| \text {. }
$$

Dual Euclidean Distance Similarly, a dual Euclidean distance can be also constructed from the difference between the elastic energies stored by two different materials for an imposed loading stress $\sigma_{0}$. In that case, the strain states read

$$
\varepsilon_{1}=\frac{\sigma_{0}}{E_{1}}, \quad \varepsilon_{2}=\frac{\sigma_{0}}{E_{2}},
$$


and the difference between elastic energies is given by

$$
w_{1}-w_{2}=\frac{1}{2}\left(\varepsilon_{1}-\varepsilon_{2}\right) \sigma_{0}=\frac{1}{2}\left(\frac{1}{E_{1}}-\frac{1}{E_{2}}\right) \sigma_{0}^{2} .
$$

It is thus possible to construct a dual Euclidean distance, expressed this time in terms of the compliances:

$$
d_{\text {dual }}\left(E_{1}, E_{2}\right)=\left|\frac{1}{E_{1}}-\frac{1}{E_{2}}\right| .
$$

Obviously, these two metrics do not give the same results since the values obtained and the units considered are not the same. With in mind the objective of formulating a unique distance between two elastic behaviors, the primal and dual Euclidean distances appear to be inappropriate. In particular, it seems important that the distance between two elastic materials cannot be expressed in the unit of stiffness or compliance in order to be invariant under inversion.

\subsection{The $f$-Euclidean Distance in the Scalar Case}

In order to overcome the dependence of the behavior law upon the stiffness (or compliance) unit, it seems necessary to express the elasticity law under a non-dimensional form. Let us consider a scalar $E_{0}>0$ which has the dimension of a stiffness, which will act as a gauge. A reduced non-dimensional elasticity law, with the gauge $E_{0}$, can thus be expressed as

$$
\frac{\sigma}{E_{0}}=\frac{E}{E_{0}} \varepsilon, \quad \varepsilon=\frac{E_{0}}{E} \frac{\sigma}{E_{0}}
$$

In that case, the sought distance should be expressed in terms of the dimensionless quantity $E / E_{0}$ (or $E_{0} / E$ ). It is important to note that the distance will consequently depend on the choice made for the gauge $E_{0}$; in particular, a given value of $E_{0}$ will generate a reduced elasticity law (14) and will lead to some family of distances denoted by $d^{E_{0}}$.

A generalized-Euclidean distance can be introduced with the gauge $E_{0}$ as:

$$
d_{f}^{E_{0}}\left(\frac{E_{1}}{E_{0}}, \frac{E_{2}}{E_{0}}\right)=\left|f\left(\frac{E_{1}}{E_{0}}\right)-f\left(\frac{E_{2}}{E_{0}}\right)\right|,
$$

where $f$ is a strictly monotone function on $\mathbb{R}^{>0}$; this allows $d_{E_{0}}^{f}$ to verify the fundamental properties of a distance function expounded in Sect. 2.1. Finally, to ensure the property of invariance by inversion

$$
d_{f}^{E_{0}}\left(\frac{E_{1}}{E_{0}}, \frac{E_{2}}{E_{0}}\right)=d_{f}^{E_{0}}\left(\frac{E_{0}}{E_{1}}, \frac{E_{0}}{E_{2}}\right),
$$

a sufficient condition for the function $f$ is that it should verify the property

$$
f(x)+f(1 / x)=\alpha, \quad \forall x \in \mathbb{R}^{>0},
$$

where $\alpha$ is a constant. It should be noted that, in Eq. (17), the "plus" sign cannot be replaced by a "minus" sign because in that case it can be shown that the function $f$ cannot be strictly monotone on $\mathbb{R}^{>0}$. 


\section{The $f$-Euclidean Distance for Elasticity Tensors: The Tensorial Case}

\subsection{Preliminaries}

First, we recall some basics of tensorial algebra, following the presentation of Moakher and Norris [8] (see also [15, 16]). Since we are investigating elastic behaviors, we are mainly concerned with fourth-order and second-order tensors in a three-dimensional Cartesian space. In particular, the tensorial elasticity law relates the second-order stress and strain tensors, respectively denoted by $\sigma$ and $\varepsilon$, through the linear relations

$$
\sigma=\mathbb{C}: \varepsilon, \quad \varepsilon=\mathbb{S}: \sigma, \quad \mathbb{C}: \mathbb{S}=\mathbb{S}: \mathbb{C}=\mathbb{I},
$$

where $\mathbb{C}$ and $\mathbb{S}$ respectively denote the fourth-order stiffness and compliance tensors, and $\mathbb{I}$ is the fourth-order identity tensor. Elasticity tensors are positive-definite and possess minor and major symmetries

$$
\begin{aligned}
& C_{i j k l}=C_{j i k l}=C_{i j l k}, \quad C_{i j k l}=C_{k l i j}, \\
& S_{i j k l}=S_{j i k l}=S_{i j l k}, \quad S_{i j k l}=S_{k l i j} .
\end{aligned}
$$

The inner product for tensors, which is needed to define the norm of a tensor, reads

$$
\langle\mathbb{A}, \mathbb{B}\rangle=A_{i j k l} B_{i j k l} .
$$

The norm $\|\mathbb{A}\|$ of a tensor $\mathbb{A}$ is then defined by

$$
\|\mathbb{A}\|=\sqrt{\langle\mathbb{A}, \mathbb{A}\rangle}=\sqrt{A_{i j k l} A_{i j k l}} .
$$

In order to facilitate some calculations, we take advantage of the Kelvin notation in elasticity (see $[15,16]$ for a comprehensive description of the Kelvin notation). Fourth-order elasticity tensors in three dimensions are equivalent to second-order tensors in six dimensions; the tensor $\mathbb{C}$ can be represented by the $6 \times 6$ matrix $\widehat{\mathbf{C}}$ defined by

$$
\widehat{\mathbf{C}}=\left(\begin{array}{cccccc}
C_{1111} & C_{1122} & C_{1133} & \sqrt{2} C_{1123} & \sqrt{2} C_{1113} & \sqrt{2} C_{1112} \\
C_{1122} & C_{2222} & C_{2233} & \sqrt{2} C_{2223} & \sqrt{2} C_{2213} & \sqrt{2} C_{2212} \\
C_{1133} & C_{2233} & C_{3333} & \sqrt{2} C_{3323} & \sqrt{2} C_{3313} & \sqrt{2} C_{3312} \\
\sqrt{2} C_{1123} & \sqrt{2} C_{2223} & \sqrt{2} C_{3323} & 2 C_{2323} & 2 C_{2313} & 2 C_{2312} \\
\sqrt{2} C_{1113} & \sqrt{2} C_{2213} & \sqrt{2} C_{3313} & 2 C_{2313} & 2 C_{1313} & 2 C_{1312} \\
\sqrt{2} C_{1112} & \sqrt{2} C_{2212} & \sqrt{2} C_{3312} & 2 C_{2312} & 2 C_{1312} & 2 C_{1212}
\end{array}\right) .
$$

Similarly, second-order symmetric tensors in three dimensions are equivalent to vectors of six dimensions. The associated stress and strain vectors denoted $\widehat{\boldsymbol{\sigma}}$ and $\widehat{\boldsymbol{\varepsilon}}$ are given by

$$
\widehat{\boldsymbol{\sigma}}=\left(\begin{array}{c}
\sigma_{11} \\
\sigma_{22} \\
\sigma_{33} \\
\sqrt{2} \sigma_{23} \\
\sqrt{2} \sigma_{13} \\
\sqrt{2} \sigma_{12}
\end{array}\right), \quad \widehat{\boldsymbol{\varepsilon}}=\left(\begin{array}{c}
\varepsilon_{11} \\
\varepsilon_{22} \\
\varepsilon_{33} \\
\sqrt{2} \varepsilon_{23} \\
\sqrt{2} \varepsilon_{13} \\
\sqrt{2} \varepsilon_{12}
\end{array}\right)
$$


With the Kelvin notation, the elasticity law can then be rewritten

$$
\widehat{\boldsymbol{\sigma}}=\widehat{\mathbf{C}} \cdot \widehat{\boldsymbol{\varepsilon}}, \quad \widehat{\boldsymbol{\varepsilon}}=\widehat{\mathbf{S}} \cdot \widehat{\boldsymbol{\sigma}}, \quad \widehat{\mathbf{C}} \cdot \widehat{\mathbf{S}}=\widehat{\mathbf{I}}_{6},
$$

where $\widehat{\mathbf{I}}_{6}$ is the $6 \times 6$ identity matrix. The norm of a tensor alternatively reads

$$
\|\mathbb{C}\|=\|\widehat{\mathbf{C}}\|=\sqrt{\widehat{\mathbf{C}}: \widehat{\mathbf{C}}}=\sqrt{\widehat{C}_{I J} \widehat{C}_{I J}}
$$

\subsection{Function of a Tensor}

In order to extend the $f$-Euclidean distance defined in the scalar case to the tensorial case, one needs to properly define functions with fourth-order tensor arguments that enjoy the major and minor symmetries. Since fourth-order elasticity tensors can be alternatively represented by second-order tensors with the Kelvin notation, it is only needed to define the function $f$ of a positive-definite $6 \times 6$ matrix. The function $f$ of a symmetric positivedefinite matrix $\widehat{\mathbf{A}}$ can be calculated in three steps:

1. Perform a diagonalization of matrix $\widehat{\mathbf{A}}$, which provides a rotation matrix $\mathbf{R}$ and a diagonal matrix $\mathbf{D}$ with the eigenvalues of $\widehat{\mathbf{A}}$ on its diagonal, such that $\widehat{\mathbf{A}}=\mathbf{R}^{\mathbf{t}} \mathbf{D R}$ (where the transpose $\mathbf{R}^{\mathrm{t}}$ of matrix $\mathbf{R}$ is defined by $R_{I J}^{\mathrm{t}}=R_{J I}$ ).

2. Write down the new diagonal matrix $\widetilde{\mathbf{D}}$ by replacing each diagonal term $\lambda_{i}$ of $\mathbf{D}$ with $f\left(\lambda_{i}\right)$.

3. Recompose $\widetilde{\mathbf{D}}$ and $\mathbf{R}$ to obtain the function $\boldsymbol{f}$ of matrix $\widehat{\mathbf{A}}$ :

$$
\boldsymbol{f}(\widehat{\mathbf{A}})=\mathbf{R}^{\mathrm{t}} \widetilde{\mathbf{D}} \mathbf{R} .
$$

It is straightforward to note that the inverse of $\widehat{\mathbf{A}}$ can be constructed as

$$
\widehat{\mathbf{A}}^{-1}=\mathbf{R}^{\mathrm{t}} \mathbf{D}^{-1} \mathbf{R} \text {. }
$$

Thus, if we assume that the scalar function $f$ considered verifies property (17), then the following result holds:

$$
\boldsymbol{f}(\widehat{\mathbf{A}})+\boldsymbol{f}\left(\widehat{\mathbf{A}}^{-1}\right)=\alpha \widehat{\mathbf{I}}_{6},
$$

where $\alpha$ is a constant.

\subsection{The Tensorial Case}

The generalized Euclidean distance in the tensorial case can be obtained as a straightforward extension of the distance defined in the scalar case. First, it is again necessary to express the elasticity law under a non-dimensional form. Let us introduce again the gauge $E_{0}>0$ (with the dimension of a stiffness) which permits the expression of the elasticity law to be written as

$$
\frac{\widehat{\boldsymbol{\sigma}}}{E_{0}}=\frac{\widehat{\mathbf{C}}}{E_{0}} \cdot \widehat{\boldsymbol{\varepsilon}}, \quad \widehat{\boldsymbol{\varepsilon}}=\left(E_{0} \widehat{\mathbf{S}}\right) \cdot \frac{\widehat{\boldsymbol{\sigma}}}{E_{0}}, \quad \frac{\widehat{\mathbf{C}}}{E_{0}} \cdot\left(E_{0} \widehat{\mathbf{S}}\right)=\widehat{\mathbf{I}}_{6} .
$$

The distance will then be expressed in terms of the dimensionless tensor $\widehat{\mathbf{C}} / E_{0}$ (or equivalently $E_{0} \widehat{\mathbf{S}}$ ). The generalized Euclidean distance thus reads

$$
d_{f}^{E_{0}}\left(\frac{\widehat{\mathbf{C}}_{1}}{E_{0}}, \frac{\widehat{\mathbf{C}}_{2}}{E_{0}}\right)=\left\|\boldsymbol{f}\left(\frac{\widehat{\mathbf{C}}_{1}}{E_{0}}\right)-\boldsymbol{f}\left(\frac{\widehat{\mathbf{C}}_{2}}{E_{0}}\right)\right\| .
$$


As mentioned above, if function $f$ verifies property (17), then Eq. (27) holds, which ensures that the distance is invariant by inversion:

$$
d_{f}^{E_{0}}\left(\frac{\widehat{\mathbf{C}}_{1}}{E_{0}}, \frac{\widehat{\mathbf{C}}_{2}}{E_{0}}\right)=d_{f}^{E_{0}}\left(\left(\frac{\widehat{\mathbf{C}}_{1}}{E_{0}}\right)^{-1},\left(\frac{\widehat{\mathbf{C}}_{2}}{E_{0}}\right)^{-1}\right) .
$$

The last ingredient is now to provide functions $f$ ensuring property (17).

\section{Closed-Form Expressions of Distances for Elasticity Tensors}

\subsection{A Subclass of Invariant-Inversion Functions}

We are looking for closed-form expressions of strictly monotone functions $f$ that verify property (17). A subclass of such functions can be constructed from arbitrary continuously differentiable functions $g$, whose properties need to be defined, such that

$$
f(x)=g(x)-g\left(\frac{1}{x}\right)+\frac{\alpha}{2}, \quad \forall x \in \mathbb{R}^{>0} .
$$

Since the desired function $f$ is strictly monotone, one has necessarily $f^{\prime}(x)>0$ or $f^{\prime}(x)<$ 0 . Since $f^{\prime}(x)=g^{\prime}(x)+g^{\prime}(1 / x) / x^{2}$, the sole restriction upon function $g$ is that is strictly monotone. The constant $\alpha$ can then be chosen arbitrarily.

It is thus possible to obtain by using Eq. (31) a large number of candidate distances by constructing function $f$ from a given strictly monotone function $g$.

\subsection{The log-Euclidean Distance}

We consider the following case

$$
\left\{\begin{aligned}
g(x) & =\frac{1}{2} \ln (x) \\
\alpha & =0
\end{aligned}\right.
$$

which leads to

$$
f(x)=\ln (x) .
$$

The associated distance for tensors, with the gauge $E_{0}$, is thus given by

$$
d_{\ln }^{E_{0}}\left(\frac{\widehat{\mathbf{C}}_{1}}{E_{0}}, \frac{\widehat{\mathbf{C}}_{2}}{E_{0}}\right)=\left\|\ln \left(\frac{\widehat{\mathbf{C}}_{1}}{E_{0}}\right)-\ln \left(\frac{\widehat{\mathbf{C}}_{2}}{E_{0}}\right)\right\| .
$$

From the definition of the logarithm of a symmetric matrix $\widehat{\mathbf{A}}$ with positive eigenvalues, it appears that

$$
\ln (\beta \widehat{\mathbf{A}})=\ln (\beta) \widehat{\mathbf{I}}_{6}+\ln (\widehat{\mathbf{A}}), \quad \forall \beta>0 .
$$

Thus, the distance $d_{\mathrm{ln}}^{E_{0}}$ given by (34) is independent of the gauge $E_{0}$ and reduces to the classical log-Euclidean distance $d_{\ln }[8,10]$ given by

$$
d_{\ln }\left(\widehat{\mathbf{C}}_{1}, \widehat{\mathbf{C}}_{2}\right)=\left\|\ln \left(\widehat{\mathbf{C}}_{1}\right)-\ln \left(\widehat{\mathbf{C}}_{2}\right)\right\|
$$




\subsection{The arctan-Euclidean Distance}

Then, we consider the case

$$
\left\{\begin{aligned}
g(x) & =\frac{1}{2} \arctan (x) \\
\alpha & =\pi / 2,
\end{aligned}\right.
$$

which leads to

$$
f(x)=\arctan (x) .
$$

The associated distance for tensors, with the gauge $E_{0}$, is called the arctan-Euclidean distance and reads

$$
d_{\arctan }^{E_{0}}\left(\frac{\widehat{\mathbf{C}}_{1}}{E_{0}}, \frac{\widehat{\mathbf{C}}_{2}}{E_{0}}\right)=\left\|\arctan \left(\frac{\widehat{\mathbf{C}}_{1}}{E_{0}}\right)-\arctan \left(\frac{\widehat{\mathbf{C}}_{2}}{E_{0}}\right)\right\| .
$$

In the scalar case, this distance has a geometrical interpretation since it corresponds to the angle between the lines of slopes $E_{1} / E_{0}$ and $E_{2} / E_{0}$ on strain-stress curves in the $\left(\varepsilon, \sigma / E_{0}\right)$ plane.

\subsection{The Power-Euclidean Distance}

We finally consider the case

$$
\left\{\begin{aligned}
g(x) & =x^{n}, \quad n \neq 0 \\
\alpha & =0
\end{aligned}\right.
$$

which leads to

$$
f(x)=x^{n}-x^{-n} .
$$

The associated family of distances for tensors, with the gauge $E_{0}$, called the powerEuclidean distances reads

$$
d_{n}^{E_{0}}\left(\frac{\widehat{\mathbf{C}}_{1}}{E_{0}}, \frac{\widehat{\mathbf{C}}_{2}}{E_{0}}\right)=\left\|\left(\frac{\widehat{\mathbf{C}}_{1}}{E_{0}}\right)^{n}-\left(\frac{\widehat{\mathbf{C}}_{2}}{E_{0}}\right)^{n}+\left(\frac{\widehat{\mathbf{C}}_{2}}{E_{0}}\right)^{-n}-\left(\frac{\widehat{\mathbf{C}}_{1}}{E_{0}}\right)^{-n}\right\|
$$

\section{Application to the Determination of the Isotropic Tensor Closest to an Arbitrary Elasticity Tensor}

\subsection{General Case}

The aim of this section is to determine the isotropic elasticity tensor closest to some arbitrary elasticity tensor by following the work of Moakher and Norris [8], Norris [11, 12]. The problem considered reduces to the minimization of the distance between the given tensor $\mathbb{C}$ and the closest isotropic tensor $\mathbb{C}_{\text {iso }}$ sought. The isotropic tensor solution $\mathbb{C}_{\text {iso }}$ of the minimization problem will thus depend on the distance considered.

A general isotropic fourth-order stiffness tensor $\mathbb{C}_{\text {iso }}$ is of the form

$$
\mathbb{C}_{\text {iso }}=3 \kappa \mathbb{J}+2 \mu \mathbb{K},
$$


where $\kappa$ and $\mu$ are respectively the bulk modulus and shear modulus; $\mathbb{I}$ and $\mathbb{K}$ are linearly independent isotropic tensors defined by

$$
\mathbb{J}=\frac{1}{3} \mathbf{I}_{3} \otimes \mathbf{I}_{3}, \quad \mathbb{K}=\mathbb{I}-\mathbb{J},
$$

where $\mathbf{I}_{3}$ is the second-order identity tensor. Following Moakher and Norris [8], it is readily seen that the function $\boldsymbol{f}$ of an isotropic fourth-order tensor $\mathbb{C}_{\text {iso }}$ is given by

$$
\boldsymbol{f}\left(\mathbb{C}_{\mathrm{iso}}\right)=f(3 \kappa) \mathbb{J}+f(2 \mu) \mathbb{K} .
$$

With the Kelvin notation, one gets

$$
\boldsymbol{f}\left(\widehat{\mathbf{C}}_{\mathrm{iso}}\right)=f(3 \kappa) \widehat{\mathbf{J}}+f(2 \mu) \widehat{\mathbf{K}} .
$$

The distance, with the gauge $E_{0}$, between some arbitrary elasticity tensor expressed in the Kelvin notation $\widehat{\mathbf{C}}$ and the closest elasticity tensor $\widehat{\mathbf{C}}_{\text {iso }}$ sought thus reads

$$
d_{E_{0}}^{f}\left(\frac{\widehat{\mathbf{C}}}{E_{0}}, \frac{\widehat{\mathbf{C}}_{\text {iso }}}{E_{0}}\right)=\left\|f\left(\frac{\widehat{\mathbf{C}}}{E_{0}}\right)-f\left(\frac{3 \kappa}{E_{0}}\right) \widehat{\mathbf{J}}-f\left(\frac{2 \mu}{E_{0}}\right) \widehat{\mathbf{K}}\right\| .
$$

The closest elasticity tensor $\widehat{\mathbf{C}}_{\text {iso }}$ minimizes the distance (47). We are thus looking for the stationarity of $d_{E_{0}}^{f}$ or equivalently $\left(d_{E_{0}}^{f}\right)^{2}$ with respect to $\kappa$ and $\mu$ :

$$
\left\{\begin{array}{l}
\frac{\partial\left(d_{E_{0}}^{f}\right)^{2}}{\partial \kappa}=0 \\
\frac{\partial\left(d_{E_{0}}^{f}\right)^{2}}{\partial \mu}=0 .
\end{array}\right.
$$

This leads to the following system:

$$
\left\{\begin{array}{l}
f\left(\frac{3 \kappa}{E_{0}}\right)=f\left(\frac{\widehat{\mathbf{C}}}{E_{0}}\right): \widehat{\mathbf{J}} \\
f\left(\frac{2 \mu}{E_{0}}\right)=\frac{1}{5} f\left(\frac{\widehat{\mathbf{C}}}{E_{0}}\right): \widehat{\mathbf{K}}
\end{array}\right.
$$

Since the scalar function $f$ is supposed to be strictly monotone, its inverse function exists (at least numerically) and is denoted $f^{-1}$. The moduli $\kappa$ and $\mu$ of the closest isotropic tensor to $\widehat{\mathbf{C}}$ for the distance $d_{E_{0}}^{f}$ are thus given by

$$
\left\{\begin{array}{l}
\kappa=\frac{E_{0}}{3} f^{-1}\left(f\left(\frac{\widehat{\mathbf{C}}}{E_{0}}\right): \widehat{\mathbf{J}}\right) \\
\mu=\frac{E_{0}}{2} f^{-1}\left(\frac{1}{5} f\left(\frac{\widehat{\mathbf{C}}}{E_{0}}\right): \widehat{\mathbf{K}}\right) .
\end{array}\right.
$$

\subsection{Example}

We investigate the determination of the closest isotropic tensor for an arbitrary distance $d_{E_{0}}^{f}$ to an a priori general stiffness tensor, the constants of which have been determined ultrasonically by François et al. [5] (see also Moakher and Norris [8]). The associated elasticity 


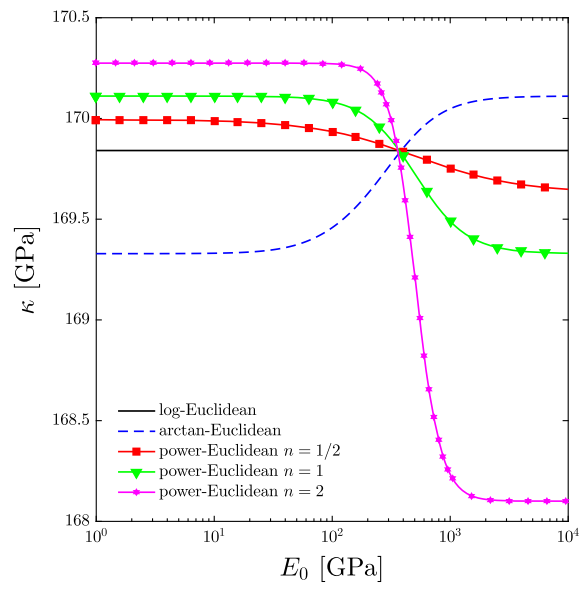

(a)

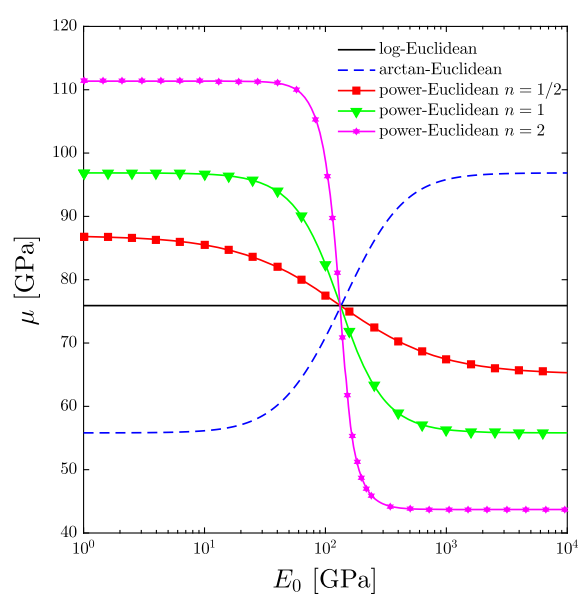

(b)

Fig. 1 Elastic moduli of closest isotropic tensors. (a) Bulk modulus $\kappa$, (b) Shear modulus $\mu$

tensor, denoted by $\widehat{\mathbf{C}}$ and expressed in the Kelvin notation, reads

$$
\widehat{\mathbf{C}}=\left(\begin{array}{cccccc}
243 & 136 & 135 & 31.1 & 73.5 & -24 \\
136 & 239 & 137 & -39.6 & 15.6 & 22.6 \\
135 & 137 & 233 & 41 & -69.3 & 4.2 \\
31.1 & -39.6 & 41 & 266 & -20 & -4 \\
73.5 & 15.6 & -69.3 & -20 & 238 & -4 \\
-24 & 22.6 & 4.2 & -4 & -4 & 260
\end{array}\right) \quad(\mathrm{GPa})
$$

The moduli $\kappa$ and $\mu$ of the closest isotropic tensors are calculated for the log-Euclidean, the arctan-Euclidean and the power-Euclidean (with $n=1 / 2,1,2$ ) distances, for a large number of gauges $E_{0}$, and are presented in Fig. 1.

As expected, the values obtained for the moduli of the closest isotropic tensors depend on (i) the type of distance considered and (ii) the value considered for the gauge (except for the log-Euclidean distance which is independent of the gauge). In both figures, all curves seem to meet at the same point. This is in fact only an effect of representation and an enlarged view would show that the curves do not exactly meet at the same point.

It is interesting to note that the values obtained for $\kappa$ and $\mu$ are bounded for each distance considered and in some cases they reach asymptotically the values obtained with the dual and primal Euclidean distances. In the limit $E_{0} \rightarrow 0$, the values for $\kappa$ and $\mu$ obtained with the power-Euclidean distance $(n=1)$ tend to the values given by the primal Euclidean distance $\left(\kappa_{\text {primal }}=170.11 \mathrm{GPa}\right.$ and $\mu_{\text {primal }}=96.87 \mathrm{GPa}$; see Moakher and Norris [8]), while the values for $\kappa$ and $\mu$ obtained with the arctan-Euclidean distance tend to the values given by the dual Euclidean distance $\left(\kappa_{\text {dual }}=169.33 \mathrm{GPa}\right.$ and $\mu_{\text {dual }}=55.81 \mathrm{GPa}$; see Moakher and Norris [8]). Conversely, in the limit $E_{0} \rightarrow+\infty$, the values for $\kappa$ and $\mu$ obtained with the power-Euclidean distance $(n=1)$ tend to the values given by the dual Euclidean distance, while the values for $\kappa$ and $\mu$ obtained with the arctan-Euclidean distance tend to the values given by the primal Euclidean distance. This means that classical Euclidean (primal and dual) distances, although not appropriate as distances for elasticity tensors, can be used to define closest isotropic tensors since the results they predict are equal to the asymptotic 
limits of those delivered by inversion-invariant (power-Euclidean or arctan-Euclidean) distances.

\subsection{Comments}

The new distances proposed in this work have been applied to the determination of the closest isotropic tensor to an arbitrary elasticity tensor. It is particularly interesting to note that all distances, except the log-Euclidean distance, lead to isotropic elastic moduli $\kappa$ and $\mu$ that depend on the gauge value. Thus it seems legitimate to wonder if the best isotropic approximation should depend on an arbitrarily chosen gauge $E_{0}$. If the independence of gauge cannot be a strict requirement in principle for a distance to be mathematically and physically acceptable, it seems reasonable to seek this independence in the application of finding the closest isotropic tensor to an arbitrary elasticity tensor. Consequently, among the functions considered in this work, it appears that the log-Euclidean distance is probably the best choice for finding the closest isotropic tensor to an arbitrary elasticity tensor.

\section{Conclusion}

Generalized Euclidean distances for elasticity have been derived. The property of invariance by inversion is preserved, which ensures that the distance between two materials is the same when represented by either their stiffness or their compliance tensors.

First, the introduction of a gauge in the tensorial elasticity law appears to be necessary in order to express the behavior law under a non-dimensional form, which leads to dimensionless elasticity tensors. Then, generalized Euclidean distances, which are based on the norm of the difference between functions of the dimensionless tensors, are introduced. A subclass of functions that achieve the property of invariance by inversion is finally proposed, which leads to new distances for tensors. The log-Euclidean distance [10] has been retrieved as a particular case, and the arctan-Euclidean as well as the power-Euclidean distances have been introduced. In particular, the arctan-Euclidean distance can be interpreted as the angle between the stress-strain curves in the scalar case.

Applied to the determination of the closest isotropic tensor to a given elasticity tensor, the new distances have permitted some interesting findings:

1. The notion of closest isotropic tensor depends drastically on the distance and the gauge considered, so it is not possible to derive a unique closest isotropic tensor to a given tensor.

2. In the limiting cases $\left(E_{0} \rightarrow 0\right.$ or $\left.E_{0} \rightarrow+\infty\right)$, the power-Euclidean (with $\left.n=1\right)$ and the arctan-Euclidean distances lead asymptotically to the predictions of the dual and primal Euclidean distances. Thus, in order to find the closest isotropic tensor to a given elasticity tensor, both dual or primal distances can in fact be used, since the results they predict are equal to the asymptotic limits predicted by invariant-inversion distances (powerEuclidean or arctan-Euclidean).

3. The log-Euclidean distance appears to be, among the functions considered here, the most interesting distance since it is independent of the gauge chosen. 


\section{References}

1. Piero, G.D.: Some properties of the set of fourth-order tensors, with application to elasticity. J. Elast. 9, 245-261 (1979)

2. Moakher, M.: Fourth-order Cartesian tensors: old and new facts, notions and applications. Q. J. Mech. Appl. Math. 61, 181-203 (2008)

3. Özarslan, E., Mareci, T.H.: Generalized diffusion tensor imaging and analytical relationships between diffusion tensor imaging and high angular resolution diffusion imaging. Magn. Reson. Med. 50, 955965 (2003)

4. Hayes, M.: A simple statical approach to the measurement of the elastic constants in anisotropic media. J. Mater. Sci. 4, 10-14 (1969)

5. François, M., Geymonat, G., Berthaud, Y.: Determination of the symmetries of an experimentally determined stiffness tensor: application to acoustic measurements. Int. J. Solids Struct. 35, 4091-4106 (1998)

6. Pokluda, J., Černý, M., Šob, M., Umeno, Y.: Ab initio calculations of mechanical properties: methods and applications. Prog. Mater. Sci. 73, 127-158 (2015)

7. Gazis, D.C., Tadjbakhsh, I., Toupin, R.A.: The elastic tensor of given symmetry nearest to an anisotropic elastic tensor. Acta Crystallogr. 16, 917-922 (1963)

8. Moakher, M., Norris, A.N.: The closest elastic tensor of arbitrary symmetry to an elasticity tensor of lower symmetry. J. Elast. 85, 215-263 (2006)

9. Moakher, M.: On the averaging of symmetric positive-definite tensors. J. Elast. 82, 273-296 (2006)

10. Arsigny, V., Fillard, P., Pennec, X., Ayache, N.: Log-Euclidean metrics for fast and simple calculus on diffusion tensors. Magn. Reson. Med. 56, 411-421 (2006)

11. Norris, A.N.: Elastic moduli approximation of higher symmetry for the acoustical properties of an anisotropic material. J. Acoust. Soc. Am. 119, 2114-2121 (2006)

12. Norris, A.: The isotropic material closest to a given anisotropic material. J. Mech. Mater. Struct. 1, 223238 (2006)

13. Bucataru, I., Slawinski, M.A.: Invariant properties for finding distance in space of elasticity tensors. J. Elast. 94, 97 (2009)

14. Deza, M.M., Deza, E.: Encyclopedia of Distances, 4th edn. Springer, Berlin Heidelberg (2016)

15. Mehrabadi, M.M., Cowin, S.C.: Eigentensors of linear anisotropic elastic materials. Q. J. Mech. Appl. Math. 43, 15-41 (1990)

16. Man, C.-S., Huang, M.: A simple explicit formula for the Voigt-Reuss-Hill average of elastic polycrystals with arbitrary crystal and texture symmetries. J. Elast. 105, 29-48 (2011) 